\title{
SPECTRAL PROPERTIES OF NORMAL OPERATORS HAVING SYMMETRIES ARISING FROM CONJUGATIONS
}

\author{
GEOFFREY R. GOODSON
}

Abstract. We study the consequences of equations such as $A B=B A$ and $A B=B A^{*}$ on the spectrum of $B$ when $A$ is a normal operator that is real or complex skew-symmetric. Our main result is a spectral pairing theorem for such operators which generalizes results about normal matrices obtained in [11]. Important tools used are the properties of conjugations and complex symmetric operators. This paper continues the study initiated in [14].

Mathematics subject classification (2010): Primary: 47B15; Secondary 47A05, 47A10.

Keywords and phrases: Normal operator, antiunitary operator, conjugation operator, complex symmetric operator.

\section{REFERENCES}

[1] R. Bhatia And P. Rosenthal, How and why to solve the operator equation $A X-X B=Y$, Bull. Lond. Math. Soc. 136 (1997), 1-21.

[2] J. B. Conway, Subnormal Operators, Research Notes in Mathematics, vol. 51. Pitman, Boston MA (1981).

[3] J. B. Conway, A Course in Operator Theory, AMS, Providence, R.I. (2000).

[4] S. R. Garcia AND M. Putinar, Complex symmetric operators and applications, Trans. Amer. Math. Soc. 358 (2005), 1285-1315.

[5] S. R. GARCiA AND M. Putinar, Complex symmetric operators and applications, II, Trans. Amer. Math. Soc. 359 (2007), 3913-3931.

[6] T. M. Gilbreath AND W. R. Wogen, Remarks on the structure of complex symmetric operators, Integral Equations and Operator Theory 59 (2007), 585-590.

[7] G. R. Goodson, A. Del Junco, M. LemańCZYK AND D. J. RudolPh, Ergodic transformations conjugate to their inverses by involution, Ergodic Theory and Dynamical Systems 16 (1996), 97-124.

[8] G. R. GOODSON AND M. LEMAŃCZYK, Transformations conjugate to their inverses have even essential values, Proc. Amer. Math. Soc. 124 (1996), 2703-2710.

[9] G. R. Goodson, The inverse-similarity problem for real orthogonal matrices, Amer. Math. Monthly. 104 (1997), 223-230.

[10] G. R. Goodson, Conjugacies between ergodic transformations and their inverses, Colloq. Math. 84/85 (2000), 185-193.

[11] G. R. GoOdson, D. Merino AND R. A. Horn, Quasi-real normal matrices and eigenvalue pairings, Linear Algebra Appl. 369 (2003), 279-294.

[12] G. R. GOODSON AND R. A. HoRn, Canonical forms for normal matrices that commute with their complex conjugate, Linear Algebra Appl. 430 (2009), 1025-1038.

[13] G. R. Goodson, On the multiplicity function of real normal operators, Operators and Matrices 2 (2008), 1-13.

[14] G. R. Goodson, Spectral doubling of normal operators and connections with antiunitary operators, Integral Equations and Operator Theory 72 (2012), 115-130.

[15] R. A. Horn and C. R. Johnson, Matrix Analysis, Cambridge University Press, New York, 1985.

[16] W. WATERHouse, The structure of alternating Hamiltonian matrices, Linear Alg. Apps. 396 (2005), $385-390$. 
[17] S. Zhu And C. G. Li, Complex symmetric weighted shifts, Trans. Amer. Math. Soc. 365 (2013), $511-530$. 